\title{
Postural Muscle Dyscoordination in Children with Cerebral Palsy
}

\author{
Jolanda C. van der Heide and Mijna Hadders-Algra \\ Department of Neurology, University of Groningen, the Netherlands
}

\begin{abstract}
The present paper gives an overview of the knowledge currently available on muscular dyscoordination underlying postural problems in children with cerebral palsy (CP). Such information is a prerequisite for developing successful therapeutic interventions in children with CP. Until now, three children with $C P$ functioning at GMFCS (Gross Motor Function Classification System) level $\mathbf{V}$ have been documented. The children totally or partially lacked direction specificity in their postural adjustments and could not sit independently for more than 3 seconds. Some children functioning at GMFCS level IV have intact direction-specific adjustments, whereas others have problems in generating consistently direction-specific adjustments. Children at GMFCS levels I to III have an intact basic level of control but have difficulties in fine-tuning the degree of postural muscle contraction to the task-specific conditions, a dysfunction more prominently present in children with bilateral spastic $C P$ than in children with spastic hemiplegia. The problems in the adaptation of the degree of muscle contraction might be the reason that children with $\mathrm{CP}$, more often than typically developing children, show an excess of antagonistic coactivation during difficult balancing tasks and a preference for cranial-caudal recruitment during
\end{abstract}

Reprint requests to: Prof. Dr. Mijna Hadders-Algra, Univ. of Groningen Medical Centre, Developmental Neurology, Hanzeplein 1, 9713 GZ Groningen, the Netherlands. e-mail: m.hadders-algra@med.rug.nl during reaching. This might imply that both stereotypies might be regarded as functional strategies to compensate for the dysfunctional capacity to modulate subtly postural activity.

\section{KEYWORDS}

spastic hemiplegia, motor development, spastic diplegia, EMG

\section{INTRODUCTION}

Children with cerebral palsy (CP) are often hampered by dysfunctional postural control (Hadders-Algra et al., 1999b; Brogren et al., 2001; Van der Heide et al., 2004). Postural control is a prerequisite for activities in daily life. In the neural control of postural adjustments two functional levels can be distinguished (Forssberg \& Hirschfeld, 1994). The first level consists of a directionspecific adjustment when equilibrium of the body is endangered. In the case of reaching, this adjustment means that the muscles on the dorsal side of the body are primarily activated when the body sways forward. The second level is involved in the fine-tuning of the direction-specific adjustment based on multisensorial afferent input from somatosensory, visual, and vestibular systems. This modulation can be achieved in various ways, for instance, by changing the order in which the agonist muscles are recruited (e.g., in a caudal-tocranial sequence or in reverse order), by modifying the size of the muscle contraction, which is reflected by the EMG-amplitude, or by altering the 
degree of antagonist activation.

Children with CP are often treated for their postural problems, but the effects of therapy are largely unknown and only partly successful or not successful at all (Mayston, 2001. Bower et al., 2001; Cioni, 2002; Washington et al., 2002). Developing successful therapeutic interventions in children with $\mathrm{CP}$ requires a better understanding of the underlying mechanisms of postural control in children with $\mathrm{CP}$. The present paper aims at giving an overview of the knowledge currently available on the muscular dyscoordination underlying postural problems in children with $\mathrm{CP}$. Special attention is paid to postural dysfunction during reaching in a sitting position.

\section{POSTURAL CONTROL IN TYPICALLY DEVELOPING CHILDREN}

The majority of studies on postural adjustments in typically developing children have been performed using external perturbations, and relatively few studies addressed postural control during self-initiated movements like reaching and walking. The studies indicated that postural adjustments during different tasks and postures, such as sitting and stance, are direction specific and variable. External perturbation experiments in a sitting position demonstrated that direction-specific postural muscle activity can already be found at the age of 1 month (Hedberg et al., 2004). Direction-specific adjustments during reaching while lying supine or sitting are present from the age at which reaching ends in successful grasping (Van der Fits et al., 1999a).

Modulation of the EMG-amplitude of the basic direction-specific adjustment in sitting emerges soon after the child has developed the skill of sitting independently and is present from 9 to 10 months onwards (Hadders-Algra et al., 1996). When the child has developed the ability to stand and walk, the capacity to modulate postural adjust- ments continues to develop, meaning that children gradually develop the ability to adapt postural activity in a subtle and energy efficient way to task-specific circumstances. One way in which the child can adapt his/her posture to the situation is by selecting from the repertoire of direction-specific adjustments the so-called en bloc adjustment (i.e., the adjustment during which all direction-specific neck and trunk muscles are activated in concert) when the risk of loosing balance is high. This explains why the en bloc strategy dominates postural control during external perturbations in sitting until the age of $2 \frac{1}{2}$ to 3 years (HaddersAlgra et al., 1998) and during walking until 7 years of age (Assaiante \& Amblard 1993; Assaiante, 1998), whereas this strategy is infrequently used during reaching in a sitting position (Van der Heide et al., 2003; Hadders-Algra, this issue).

The participation of antagonists - muscles that antagonize the function of the primarily activated muscles - in the postural adjustment mainly occurs when postural balance is at stake, for instance when children learn a new skill such as standing or walking independently (Forssberg \& Nashner 1982. Berger et al., 1984). In general, antagonistic postural muscles are rarely recruited during sitting tasks. There are two transient exceptions to this rule. First, infants aged 8 to 18 months show a high rate of neck flexor activation during reaching - a finding that underscores the need for head stabilization in space for such individuals during this task (Van der Fits et al., 1999b). Second, children aged 9 months to 2 years frequently recruit antagonistic, dorsally located neck, trunk, and leg muscles during perturbations inducing a backward body sway, a situation which really challenges balance (Hadders-Algra et al., 1998). In standing, antagonistic activation is present at least until the age of 5 years (Forssberg \& Nashner, 1982). When brought into play, the antagonists usually are not activated synchronously with the agonistic muscle but rather only after a delay of at least $40 \mathrm{~ms}$. 
During infancy, the temporal organization of muscle activity within direction-specific adjustments is highly variable (Hirschfeld \& Forssberg, 1994. Hadders-Algra et al., 1996. Van der Fits et al., 1999b. Hedberg et al., unpublished; Washington et al., 2004). Nevertheless, during early infancy a mild preference for a top-down recruitment order can be observed (Hadders-Algra et al., 1996). In postural adjustments during external perturbations in sitting, the mild dominance of top-down recruitment changes around the end of the first year into a bottom-up recruitment (Hadders-Algra et al., 1996. Washington et al., 2004). The latter can be observed particularly when there is a high risk of loosing balance, i.e., during perturbations in stance (Shumway-Cook \& Woollacott, 1985. Sundermier et al., 2001). The recruitment order of directionspecific muscles during reaching while sitting remains highly variable throughout childhood. Also here general developmental trends can be distinguished: at early sitting age, postural muscles are most often recruited in a bottom-up fashion, whereas from 5 years onwards a mild preference for top-down recruitment emerges (Van der Fits et al., 1999a. Van der Heide et al., 2003).

The presence of anticipatory postural activitydefined as postural activity preceding the focal, voluntary movement-is dependent on task, position, and age. Anticipatory postural activity during reaching while sitting occurs consistently in infants only of 15 to 18 months (Van der Fits et al., 1999b. Van der Heide et al., 2003), but during most standing and walking, tasks anticipatory postural adjustments are consistently present beyond the age of 18 months (Forssberg \& Nashner, 1982. Haas et al., 1989. Assaiante et al., 2000). A task during which no consistent anticipatory postural adjustments have been found in children aged 4 to 14 years was an arm-raising task during stance (Riach \& Hayes, 1990).

The development of the subtle modulation of EMG amplitude shows a protracted course. The capacity to modulate EMG amplitude based on proprioceptive information emerges around 9 to 10 months (Hadders-Algra et al., 1996. Van der Fits et al., 1999h). The postural muscle that forms the focus of modulation - the postural muscle showing the most marked amplitude modulation-varies with age and task. For instance, in adults who reach while sitting, the neck muscles are the focus of amplitude modulation. During childhood, however, none of the postural muscles has the primacy for amplitude modulation during reaching in sitting (Van der Heide et al., 2003). In stance, caudally located muscles usually are the focus of task-dependent modulation (Woollacott et al., 1987. Berger et al., 1995).

\section{POSTURAL DYSCOORDINATION IN CHILDREN WITH CEREBRAL PALSY}

In the majority of children with $\mathrm{CP}$, the basic level of postural control is intact. Only children with severe forms of $\mathrm{CP}$-children functioning at levels IV or V of the GMFCS (Gross Motor Function Classification System. Palisano et al., 1997)—have problems in generating direction-specific adjustments (Hadders-Algra et al., 1999a. Brogren et al., 2001). Postural abilities in these severely affected children have been tested in a sitting position only. Until now, three children with $C P$ functioning at GMFCS level $\mathrm{V}$ have been documented (Hadders-Algra et al., 1999a,b. Van der Heide et al., 2004). Two totally lacked direction specificity in their postural adjustments and were unable to sit independently at the age of 4 years. The third child had direction-specific activity in neck and trunk muscles but was unable to generate consistently direction-specific activity in the leg muscles. At the age of 8 years, she could sit without help for less than 3 seconds. Data on postural adjustments in children with $\mathrm{CP}$ functioning at GMFCS level IV are available for eight children. Five had difficulties in producing consistently direction specific activity at the level 
of the neck or leg muscles. The other three children were able to generate consistently direction specific adjustments (Brogren et al., 2001. Van der Heide et al., 2004).

All children with CP show alterations in the second level of postural control-in the recruit-ment of antagonistic postural muscles, in the temporal organization and EMG-amplitude modu-lation. Most studies addressing postural control in children with $\mathrm{CP}$ used the external perturbation paradigm. These studies showed that children with $\mathrm{CP}$, aged $1 \frac{1}{2}$ to 11 years, show differences in recruitment order, differences in latencies to onset of postural muscle activation, and a higher level of antagonistic co-activation than typically developing children do (Nashner et al., 1983. Brogren et al., 1996. Woollacott et al., 1998). Children with CP also have deviances in the modulation of EMG-amplitude to task-specific circumstances (Brogren et al., 2001). A remarkable finding was that this deficit in EMGamplitude modulation was attenuated when the children were allowed to sit in their usual crouched sitting position (Brogren et al., 2001).

In the following section, we will zoom in on our own research on postural control during reaching in a sitting position in children with $\mathrm{CP}$.

\section{Postural adjustments during reaching while sitting}

Hadders-Algra et al. (1999b) and Van der Heide et al. (2004) focused on the development of postural adjustments during voluntary reaching in children with CP. Postural control during reaching was studied longitudinally in 5 infants with spastic hemiplegia (SH) and in 2 infants with severe bilateral CP (Bi-CP) between the ages of 4 and 18 months (Hadders-Algra et al., 1999b) and crosssectionally in 34 children with SH and 24 children with Bi-CP, aged 2 to 11 years (Van der Heide et al., 2004).

The results of the studies indicated that most children with $\mathrm{CP}$ showed variable postural muscle activity at all ages. This variability was reflected by the virtual absence of the en bloc pattern from the age of 2 years onwards. In only some children with severe $\mathrm{CP}$, reaching movements were accompanied consistently by slow and weak modulations of tonic activity of the postural muscles, whereas a few others with severe CP strongly and consistently activated most postural muscles during reaching. The abnormalities in the postural activation patterns of children with severe CP corresponded with the muscle tone of their neck and trunk muscles. Children with variable postural muscle activation had a normal tone of neck and trunk muscles, whereas children with consistently weak or consistently strong postural activity had hypotonia and hypertonia of their neck and trunk muscles, respectively.

Our studies also demonstrated that children with $\mathrm{CP}$, who are able to reach, have difficulties mainly at the second level of postural control during reaching in a sitting position. The children had a temporal organization of the adjustments that differed from that of typically developing children and had dysfunctions in the ability to modulate EMG-amplitude to task-specific circumstances. Remarkably, children with $\mathrm{CP}$, just like typically developing children, show hardly any antagonistic co-activation in postural muscles during reaching in a sitting position. Thus, children with $\mathrm{CP}$ do not exhibit an excess of antagonistic co-activation during this postural task, whereas they do in conditions in which balance is more threatened, such as during external perturbations. This means that antagonistic co-activation in children with $\mathrm{CP}$ is not a hard-wired deficit but rather can be regarded as a functional adaptation to, for instance, tasks with a high degree of balancing difficulty.

In contrast to typically developing children, children with $\mathrm{CP}$ showed a strong preference for a cranial-caudal recruitment order of the directionspecific postural muscles (Fig. 1). The dominance for top-down recruitment was brought about by a 


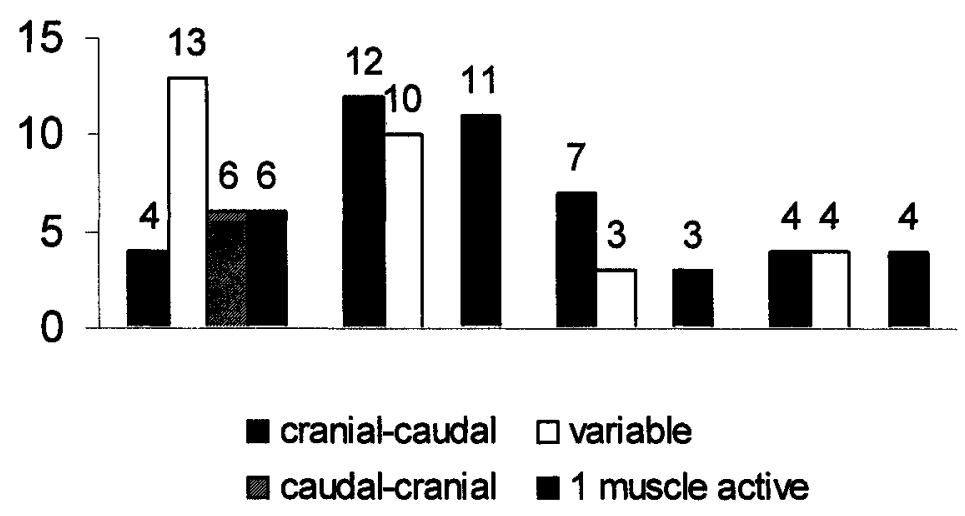

Fig. 1: Recruitment order of direction specific muscles in typically developing children and children with mild, moderate, or severe forms of CP. The severity of the motor disorder was determined during a neurological examination. Prevailing recruitment order was determined based on the mean latencies after prime mover onset per child. Crania-caudal recruitment: trunk muscle recruited $>100 \mathrm{~ms}$ later than neck muscle. caudal-cranial recruitment: neck muscle recruited $>100 \mathrm{~ms}$ later than trunk muscle. variable recruitment order: neck and trunk muscles recruited with a mean delay of $\leq 100 \mathrm{~ms}$. 1 muscle active: either neck extensor or trunk extensor was activated in isolation precluding the calculation of a recruitment order.

slow recruitment of the trunk muscles and a fast recruitment of the neck muscles. The latter was true in particular for children with a mild or moderate form of $\mathrm{CP}$. We wondered whether the prominent preference for top-down recruitment could be regarded as a dysfunction or as a functional strategy to cope with task-specific circumstances ( $c f$. Latash \& Anson, 1996).

Arguments in favor of both explanations are present. Arguments for the assumption that the dominance of top-down recruitment can be regarded as a sign of dysfunction are the findings that the preference for cranio-caudal muscle activation was related to a worse mobility score on the Pediatric Evaluation of Disability Inventory (PEDI. Custers et al., 2002), and to the presence of a severe brain lesion on the neonatal ultrasound scan in the children with SH. The argument that the prominent presence of top-down recruitment can be regarded as a functional strategy is the finding that this strategy was more often seen in children with mild or moderate $\mathrm{CP}$ than in children with severe CP. The top-down organization of the postural adjustments might be a reflection that head stabilization in space is a major goal of postural control (cf. Pozzo et al., 1990).

The difficulties in EMG-amplitude modulation of the children with CP consisted of problems in adapting the EMG amplitude according to taskspecific circumstances, such as arm movement velocity, initial head, trunk and pelvis position, and the presence of a heavy bracelet. These deficits were more profound in children with $\mathrm{Bi}$ CP than in children with SH. An inability to modulate EMG amplitude was associated with a significantly worse quality of reaching movements (Van der Heide et al., under review) andparticularly in children with $\mathrm{Bi}-\mathrm{CP}$ - to somewhat worse scores on the PEDI, i.e., the capacity to perform activities of daily life. 


\section{CONCLUDING REMARKS}

Conceivably, in children functioning at GMFCS level $\mathrm{V}$, the basic level of postural control is lacking totally or partially, meaning that the management of these children should focus on the provision of adequate postural support and not on the achievement of postural milestones. Children functioning at GMFCS level IV have a totally or partially intact basic level of control, and children at GMFCS levels I to III all have an appropriate access to direction-specific postural adjustments.

Children with $\mathrm{CP}$, in particular, have difficulties in fine-tuning the degree of postural muscle contraction to the task specific conditions, a dysfunction that is more prominently present in children with bilateral spastic $\mathrm{CP}$ than in children with spastic hemiplegia. The problems in the adaptation of the degree of muscle contraction might be the reason that children with $\mathrm{CP}$, more often than typically developing children, show an excess of antagonistic co-activation during difficult balancing tasks and a preference for cranial-caudal recruitment during reaching. This situation might imply that both stereotypies might be regarded as functional strategies to compensate for the dys-functional capacity to modulate postural activity subtly. This in turn, might suggest that guidance of children with mild to moderate forms of CP should not focus on the reduction of co-activation or top-down recruitment but rather on balancing exercises, in which children can practice varying degrees of postural control.

\section{ACKNOWLEDGMENTS}

Mr. L.A. van Eykern, Ms. A.A. Kingmabalkema, and Dr. J.J.A.M. Schaap are kindly acknowledged for their skilful technical assistance. The study was supported by the Johanna Kinderfonds (Grant number 19990021), the Dr. W.M. Phelps-stiching voor spastici (grant number 99.058), the gratama stichting/groninger universiteits fonds and the algemeen welzijnsfonds voor geestelijk en lichamelijk gehandicapten.

\section{REFERENCES}

Assaiante C, Amblard B. 1993. Ontogenesis of head stabilization in space during locomotion in children: influence of visual cues. Exp Brian Res 93: 499-515.

Assaiante C. 1998. Development of locomotor balance control in healthy children. Neurosci Biobehav $\operatorname{Rev} 22: 527-532$.

Assaiante C, Woollacott M, Amblard B. 2000. Development of postural adjustment during gait initiation: kinematic and EMG analysis. J Mot Behav 32: 211-226.

Berger W, Altenmüller E, Dietz V. 1984. Normal and impaired development of children's gait. Hum Neurobiol 3: 163-170.

Berger W, Trippel M, Assaiante C, Zijlstra W, Dietz V. 1995. Developmental aspects of equilibrium control during stance: a kinematic and EMG study. Gait Posture 3: 149-155.

Bower E, Michell D, Burnett M, Campbell MJ, McLellan DL. 2001. Randomized controlled trial of physiotherapy in 56 children with cerebral palsy followed for 18 months. Dev Med Child Neurol 43: 4-15.

Brogren E, Hadders-Algra M, Forssberg H. 1996, Postural control in children with spastic diplegia: muscle activity during perturbations in sitting. Dev Med Child Neurol 38: 379-388.

Brogren E, Forssberg H, Hadders-Algra M. 2001. Influence of two different sitting positions on postural adjustments in children with spastic diplegia. Dev Med Child Neurol 43: 534-546.

Cioni G. 2002. Natural history and treatment of disabilities. Dev Med Child Neurol 44: 651-651.

Custers JW, Wassenberg-Severijnen JE, Van der Net J, Vermeer A, Hart HT, Helders PJ. 2002. Dutch adaptation and content validity of the 'Pediatric Evaluation Of Disability Inventory (PEDI)'. Disabil Rehabil 24: 250-258.

Forssberg H, Nashner LM 1982. Ontogenetic development of postural control in man: adaptation to altered support and visual conditions during stance. J Neurosci 2: 545-552. 
Forssberg H, Hirschfeld H. 1994. Postural adjustments in sitting humans following external perturbations: muscle activity and kinematics. Exp Brain Res 97: 515-527.

Haas G, Diener HC, Rapp H, Dichgans J. 1989. Development of feedback and feedforward control of upright stance. Dev Med Child Neurol 31: 481-488.

Hadders-Algra M. 2005. Development of postural control during the first 18 months of life. Neural Plast, this issue.

Hadders-Algra M, Brogren E, Forssberg H. 1996. Ontogeny of postural adjustments during sitting in infancy: variation, selection and modulation. $J$ Physiol 493: 273-288.

Hadders-Algra M, Brogren E, Forssberg H. 1998. Postural adjustments during sitting at preschool age: presence of a transient toddling phase. Dev Med Child Neurol 40: 436-447.

Hadders-Algra $M$, Brogren E, Katz-Salamon M, Forssberg H. 1999a. Periventricular leukomalacia and preterm birth have a different detrimental effect on postural adjustments. Brain 122: 727-740.

Hadders-Algra M, Van der Fits IBM, Stremmelaar EF, Touwen BCL 1999b. Development of postural adjustments during reaching in infants with CP. Dev Med Child Neurol 41: 766-776.

Hedberg $\AA$, Forssberg H, Hadders-Algra M. 2004. Postural adjustments due to external perturbations during sitting in 1-month-old infants: evidence for the innate origin of direction specificity. Exp Brain Res 157: 10-17.

Latash ML, Anson JG. 1996. What are "normal movements" in atypical populations? Behav Brain Sci 1996. 19: 55-106.

Mayston MJ. 2001. People with cerebral palsy: effects of and perspectives for therapy. Neural Plast 8: 51-69.

Nashner LM, Sumway-Cook A, Marin O. 1983. Stance posture control in select groups of children with cerebral palsy: deficits in sensory organization and muscular coordination Exp Brain Res 49: 393-409.

Palisano R, Rosenbaum P, Walter S, Russell D, Wood E, Galuppi B. 1997. Development and reliability of a system to classify gross motor function in children with cerebral palsy. Dev Med Child Neurol 39: 214-223.

Pozzo T, Berthoz A, Lefort L. 1990. Head stabilization during various locomotor tasks in humans. I. Normal subjects. Exp Brain Res 82: 97-106.

Riach CL, Hayes KC. 1990. Anticipatory postural control in children. J Mot Behav 22: 250-256.

Shumway-Cook A, Woollacott MH. 1985. The growth of stability: postural control from a developmental perspective. J Mot Behav 17:131147.

Sundermier L, Woollacott $\mathrm{MH}$, Roncesvalles N, Jensen J. 2001. The development of balance control in children: comparisons of EMG and kinetic variables and chronological and developmental groupings Exp Brain Res 136: 340-350.

van der Fits IB, Klip AW, van Eykern LA, HaddersAlgra M. 1999a. Postural adjustments during spontaneous and goal-directed arm movements in the first half year of life. Behav Brain Res 106: 75-90.

Van der Fits IB, Otten E, Klip AW, Van Eykern LA, Hadders-Algra M. 1999b. The development of postural adjustments during reaching in 6- to 18 month-old infants. Evidence for two transitions. Exp Brain Res 126: 517-528.

van der Heide JC, Otten B, van Eykern LA, HaddersAlgra M. 2003. Development of postural adjustments during reaching in sitting children. Exp Brain Res 151: 32-45.

van der Heide JC, Begeer C, Fock JM, Otten B, Stremmelaar E, van Eykern LA, et al. 2004. Postural control during reaching in preterm children with cerebral palsy. Dev Med Child Neurol 46: 253-266.

Washington K, Deitz JC, White OR, Schwartz IS 2002. The effects of a contoured foam seat on postural alignment and upper-extremity function in infants with neuromotor impairments. Phys Ther 82: 1064-1076.

Washington K, Shumway-Cook A, Price R, Ciol M, Kartin D. 2004. Muscle responses tot seated perturbations for typically developing infants and those at risk for motor delays. Dev Med Child Neurol 46: 681-688.

Woollacott M, Burtner P, Jensen J, Jasiewicz J, Roncesvalles N, Sveistrup H. 1998. Development of postural responses during standing in healthy children and in children with spastic diplegia. Neurosci Biobehav Rev 22: 583-589.

Woollacott M, Debu B, Mowatt M. 1987. Neuromuscular control of posture in the infant and child: is vision dominant? J Mot Behav 19: 167-186. 

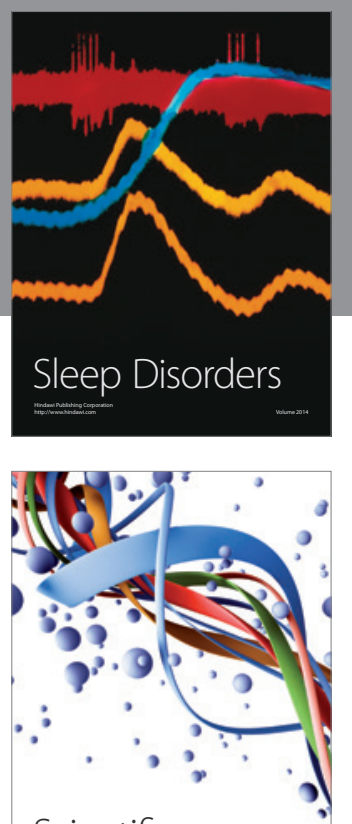

Scientifica
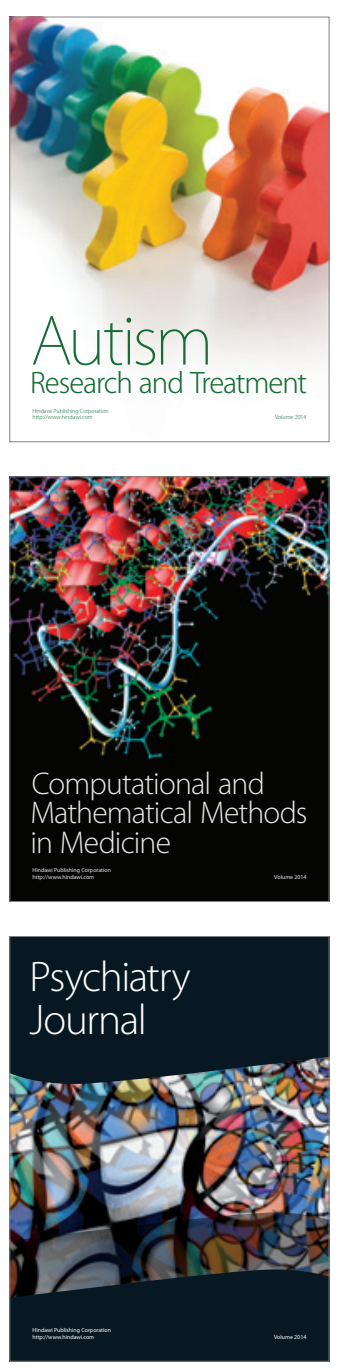
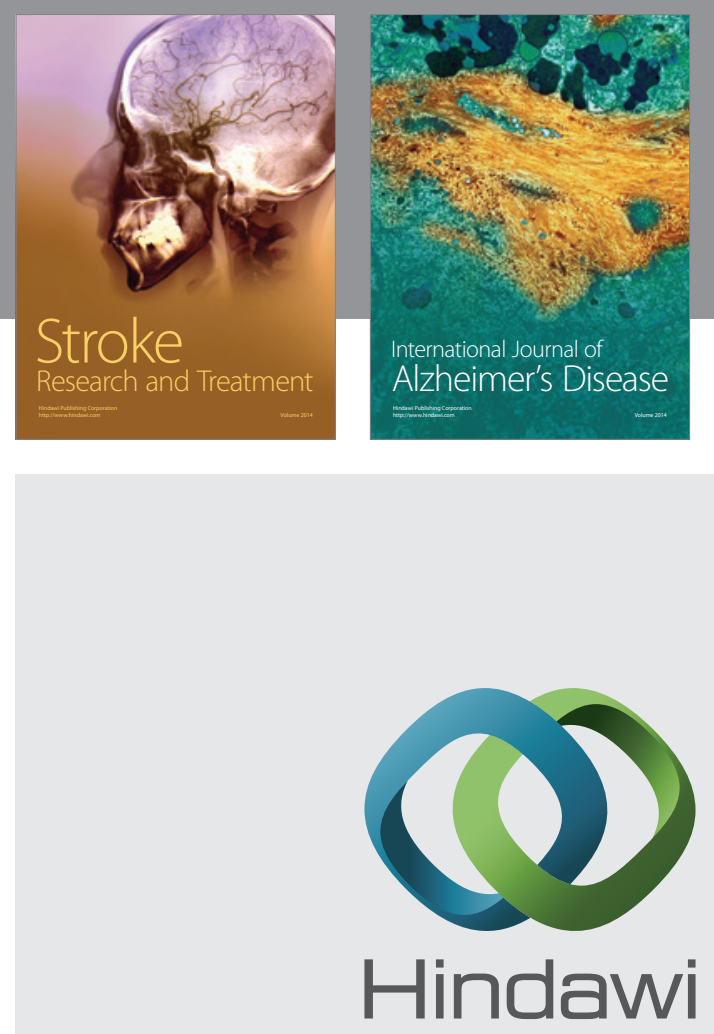

Submit your manuscripts at

http://www.hindawi.com
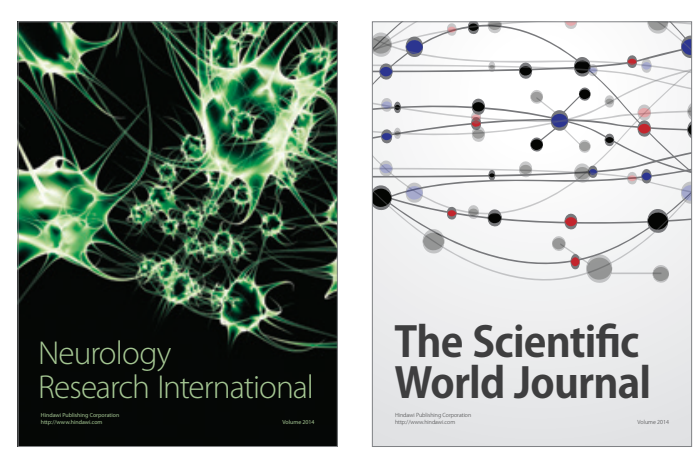

The Scientific World Journal

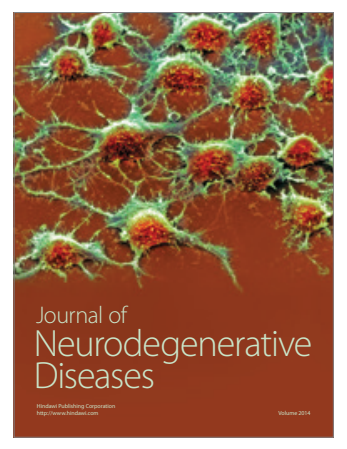

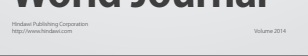

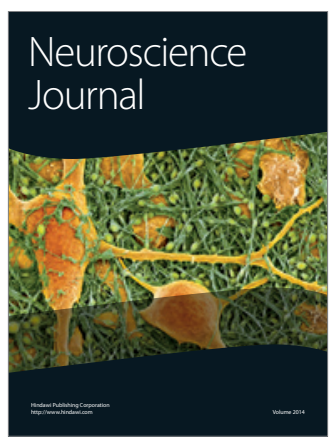

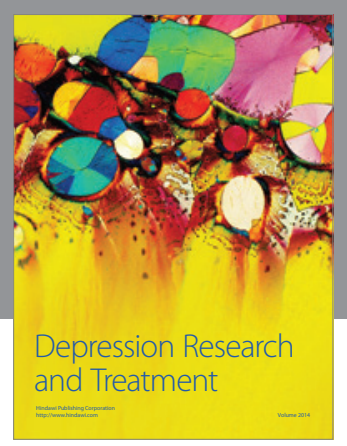
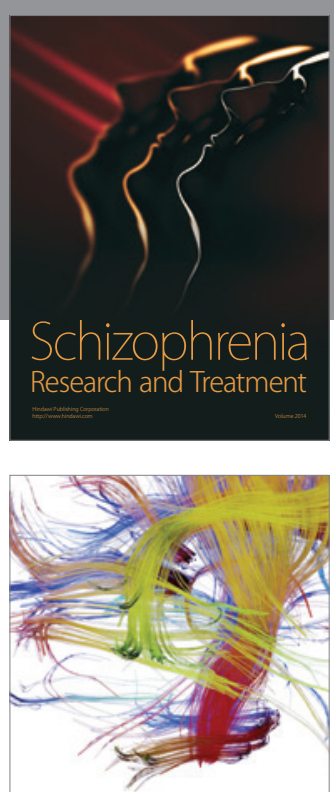

Brain Science

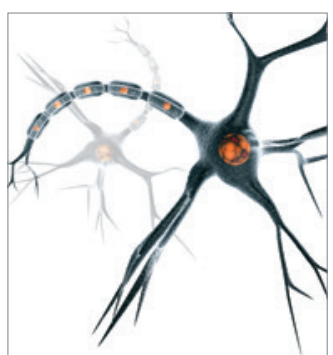

Neural Plasticity
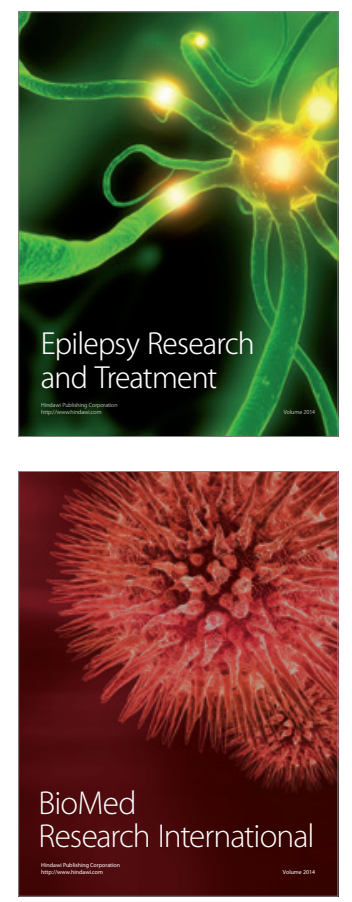

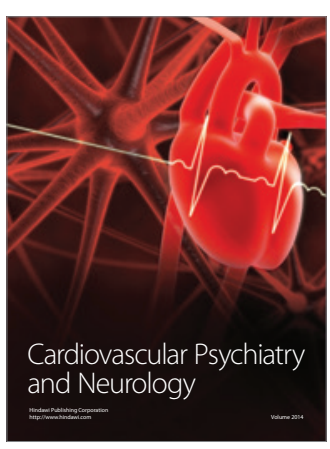

Parkinson's

Disease
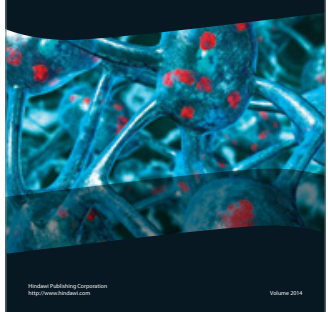